\title{
Macroscopic chiral symmetry breaking in monolayers of achiral nonconvex platelets
}

DOI:

10.1039/C7SM01840A

\section{Document Version}

Accepted author manuscript

Link to publication record in Manchester Research Explorer

\section{Citation for published version (APA):}

Pakalidou, N., Cheung, D. L., Masters, A., \& Avendano , C. (2017). Macroscopic chiral symmetry breaking in monolayers of achiral nonconvex platelets. Soft Matter, 13, 8618-8624. https://doi.org/10.1039/C7SM01840A

\section{Published in:}

Soft Matter

\section{Citing this paper}

Please note that where the full-text provided on Manchester Research Explorer is the Author Accepted Manuscript or Proof version this may differ from the final Published version. If citing, it is advised that you check and use the publisher's definitive version.

\section{General rights}

Copyright and moral rights for the publications made accessible in the Research Explorer are retained by the authors and/or other copyright owners and it is a condition of accessing publications that users recognise and abide by the legal requirements associated with these rights.

\section{Takedown policy}

If you believe that this document breaches copyright please refer to the University of Manchester's Takedown Procedures [http://man.ac.uk/04Y6Bo] or contact uml.scholarlycommunications@manchester.ac.uk providing relevant details, so we can investigate your claim.

\section{OPEN ACCESS}




\title{
Journal Name
}

\section{ARTICLE TYPE}

Cite this: DOI: $10.1039 / x x x x x x x x x x$

\section{Macroscopic chiral symmetry breaking in monolayers of achiral nonconvex platelets ${ }^{\dagger}$}

\author{
Nikoletta Pakalidou, ${ }^{a}$ David L. Cheung, ${ }^{b}$ Andrew J. Masters,${ }^{a}$ and Carlos Avendaño*a
}

Received Date

Accepted Date

DOI: $10.1039 / x x x x x x x x x x$

www.rsc.org/journalname
The fabrication of chiral structures using achiral building blocks is a fundamental problem that remains a challenge in materials science. In this work we present a molecular dynamics simulation study of nonconvex polygonal platelets interacting via soft-repulsive interactions confined in two-dimensional space. These particle models are designed to promote even at moderate densities a natural offset displacement between the edges of neighbouring particles. In particular we demonstrate that nonconvex platelets exhibit macroscopic chiral symmetry breaking when the symmetry of the particles equals (or is multiple of) the number of nearest neighbours in the condensed crystalline phase, corresponding to the situation of platelets with 4-, 6-, and 12-fold symmetries.

\section{Introduction}

Lord Kelvin introduced the term chirality more than 100 years ago 3 to describe the symmetry of geometrical objects and set of points in space ${ }^{1,2}$. Chirality is a fundamental concept central in a broad range of applications such as development of pharmaceuticals ${ }^{3}$, chiral recognition and sensing ${ }^{4,5}$, enantioselective separation and catalysis $^{6,7}$, negative refraction of elastic waves ${ }^{8}$, and in the design of optical devices ${ }^{9}$. Historically, the term chirality has been associated mainly to the field of stereochemistry, but it is also used to describe the symmetric properties of structured materials in the nano, colloidal, and macroscopic scales.

Selecting building blocks of suitable symmetry and interactions can be used to break the chiral symmetry of the system forming enantiomeric clusters or domains, i.e. the system exhibits local chiral symmetry breaking (CSB). Macroscopic CSB emerges when a single enantiomer prevails across the entire system. Chiral nano and colloidal building blocks can be used to self-assemble chiral materials across the mesoscale ${ }^{10,11}$. However, it is of fundamental and practical interest the design of chiral structures using achiral building blocks interacting purely by excluded volume interactions. ${ }^{11-18}$ Triangular platelets confined in two-dimensional monolayers highlight the subtleties and challenges of assembling chiral structures based only on the shape of achiral particles. Zhao and co-workers ${ }^{12}$, for example, reported the self-assembly of lithographic achiral triangles confined in two-dimensional (2D)

a School Chemical Engineering and Analytical Science, The University of Manchester, Sackville street, Manchester, M3 9PL, UK; E-mail: carlos.avendano@manchester.ac.uk ${ }^{b}$ School of Chemistry, National University of Ireland, Galway, University Road, Galway, Ireland

$\dagger$ Electronic Supplementary Information (ESI) available: [details of any supplementary information available should be included here]. See DOI: 10.1039/b000000x/ monolayers, observing the formation of a triatic phase characterised by three-fold quasi-long range orientational order. At high particle concentrations, this triatic phase exhibits a local CSB in which neighbouring particles around a central particle, rather than being aligned face-to-face with their apothems sharing the same axis, exhibit an offset angular displacement $\theta$ with either positive or negative directions resulting in different enantioners. The authors proposed an explanation of this local CSB in terms of rotational entropy arguments. Later on, Carmichael and Shell performed a detailed computer simulation study of monolayers of triangular platelets with rounded corners ${ }^{13}$. In particular, the authors found that corner rounding in itself, which is present in the experimental particles of Zhao and co-workers ${ }^{12}$, promotes local CSB. Gantapara and co-workers ${ }^{19}$ revisited the phase diagram of perfect equilateral triangles ${ }^{13,20}$. The authors observed the formation of a triatic phase similar to the one observed in the experimental study of Zhao and co-workers ${ }^{12}$, but there was not evidence of local CSB in the triatic phase at moderate densities. However, these authors found that at high densities equilateral triangles form a nearly perfect triangular crystal phase with the particles experiencing a cooperative orientational displacement in a common direction from the perfect crystal lattice leading to the spontaneous formation of small chiral holes located at the positions where the vertices of the particles meet. Surprisingly, the handedness of these holes extends long distances resulting in a unique example of entropy-driven macroscopic CSB in a system of achiral particles ${ }^{19}$.

In this work, we study the self-assembly monolayers of nonconvex curved polygonal platelets, i.e. achiral polygons with curved sides. This model is designed to promote even at moderate densities a natural offset displacement between the edges of neigh- 
bouring particles. In particular we demonstrate that when the symmetry of the nonconvex platelets equals the number of nearest neighbours per particle, the system experiences macroscopic CSB driven purely by packing effects.

\section{Methodology}

The polygonal platelets are represented as a collection of $N_{b}$ spheres of diameter $\sigma$ forming a planar rigid ring interacting via the Weeks-Chandler-Andersen (WCA) soft-repulsive potential ${ }^{21}$. Note that the large internal cavity of the particles is not relevant in $2 \mathrm{D}$, thus the rings represent planar nonconvex platelets of area $A_{p}$ (see the Appendix for more details of the particle model). We have explored polygonal platelets with 3, 4, 5 and 6-fold symmetries. Less extensive simulations for system with 7-, 8-, and 12fold symmetries have also been carried out. The number of beads $N_{b}$ used for each platelet is such that the length of the edges is the same for all platelets (13 beads per side). The curvature of the platelets is controlled by shifting the beads of the sides, originally separated by a distance $\sigma / 2$, towards the centre of the particle (keeping the beads forming the vertices fixed) according to a quadratic function $y=y(x ; n, k)$, where $n$ is the symmetry of the platelets and $k$ is the degree of curvature. The results presented in this study correspond to platelets with a curvature of $k=1 / 3$, which corresponds the central beads of each edge being shifted towards the centre of the polygons by $1 / 3$ of the apothem. The dynamics of the particles is constrained to a rectangular region of area $A=L_{x} L_{y}$, where $L_{x}$ and $L_{y}$ are the lengths of the simulation cell. Thermodynamic properties are expressed in dimensionless units using LJ parameters where the temperature, density, packing fraction, pressure, and time are given by: $T^{*}=k_{B} T / \varepsilon$, $\rho^{*}=N \sigma^{2} / A, \phi=A_{p} N / A, p^{*}=p \sigma^{2} / \varepsilon$, and $t^{*}=t /\left(m \sigma^{2} / \varepsilon\right)^{1 / 2}$, respectively, where $T$ is the absolute temperature, $k_{B}$ is the Boltzmann's constant, $\varepsilon$ is the LJ well-depth, $N$ is the total number of platelets, $p$ is the absolute pressure, and $m$ is the mass of the beads. The simulation details are the same as previously used for the case of rigid planar colloidal rings in three dimensional space ${ }^{22-27}$. Molecular dynamics (MD) simulations are performed using LAMMPS ${ }^{23}$ in the isothermal-isobaric (NPT) ensemble with periodic boundary conditions in the $x$ and $y$ directions. Simulations using the isothermal-isostress $N \sigma_{p} T$ ensemble are also used in crystalline phases ${ }^{28}$. The velocity Verlet algorithm is used to integrate the Newton's equations of motions using a time step of $\Delta t^{*} \sim 0.003$. The temperature and pressure are controlled using a Nosè-Hoover thermostat and barostat ${ }^{24-26}$. The temperature is fixed at $T^{*}=1.25$ to approximate the hard-sphere behaviour of the spherical beads used in the model ${ }^{29}$. Extensive simulations for all polygons are carried out using systems comprising $N=1024$ platelets, but simulations of systems with 529, 2025, 4900 platelets have also been used to confirm that the formation of some ordered phases is not influenced by system size effects. Correlation functions for selected states are calculated using systems of $N=9216$ platelets. Close-packed configurations of the different nonconvex platelets are determined using a pressure annealing Monte Carlo method using cells with 4 particles ${ }^{27}$. In these simulations, the beads of the polygons are modelled as hard-spheres of diameter $\sigma$. For each system, 50 sequential com- pressions with different initial configurations are carried out and final structures are saved for post-processing analysis.

The order of the systems is quantified using several local and global order parameters. Global bond-order parameters $\Psi_{l}$ are used to quantify $l$-fold symmetry of the system ${ }^{30}$ and are defined as

$$
\Psi_{l}=\frac{1}{N}\left|\sum_{j=1}^{N} \psi_{l, j}\right|=\frac{1}{N}\left|\sum_{j=1}^{N} \frac{1}{N_{j}} \sum_{k=1}^{N_{j}} \exp \left(i l \theta_{j k}\right)\right|,
$$

where $\psi_{l, j}$ is the local bond-order parameter of particle $j, N_{j}$ is the number of nearest neighbours of particle $j$ determined using a Voronoi tessellation algorithm ${ }^{31}$, and $\theta_{j k}$ is the angle made by the bond between particle $j$ and its nearest neighbour $k$ with respect to an arbitrary axis. Orientational order is analysed using the global orientational order parameter 32,33

$$
\Phi_{n}=\frac{1}{N}\left|\sum_{j=1}^{N} \exp \left[\operatorname{in}\left(\theta_{j} \bmod (2 \pi / n)\right)\right]\right|,
$$

where $n$ is the symmetry of the platelets. The appearance chiral symmetry breaking is quantified using a chiral order parameter $X_{c}$ defined in this work as

$$
X_{c}=\frac{1}{N} \sum_{j=1}^{N} \chi_{c, j}=\frac{1}{N} \sum_{j=1}^{N} \frac{1}{N_{j}}\left[N_{j}^{+}-N_{j}^{-}\right],
$$

where $\chi_{c, j}$ is the local chiral order parameter of particle $j$, and $N_{j}^{+}$and $N_{j}^{-}$are the number of nearest neighbours of particle $j$ adopting clockwise or anticlockwise offset angular displacements of their edges, respectively. Note that this parameter can take the limiting values of $X_{c} \sim-1$ (macroscopic left handed CSB), $X_{c} \sim$ 1 (macroscopic right handed CSB), and $X_{c} \sim 0$ (achiral/racemic structures).

\section{Results}

We discuss first the results for the phase behaviour of hexagonal platelets obtained from compression runs, which are presented in Figure 1(a). Expansion runs starting from a perfect crystal led to equivalent results. On sequential compression using NPT MD simulations the system exhibits a first-order phase transition from an isotropic (Iso) state $\left(\phi=0.618, \Psi_{6}=0.302, \Phi_{6}=0.088\right)$ to a random hexagonal (RHx) state $\left(\phi=0.632, \Psi_{6}=0.701, \Phi_{6}=0.177\right)$ at $p^{*} \sim 0.113$. This transition is characterised by a discontinuity of the hexagonal bond-order parameter $\Psi_{6}$, with only a small increment of orientational order parameter $\Phi_{6}$ indicating the system remains orientationally disordered. The order parameter $\Psi_{4}$ remains negligible confirming the structure is indeed hexagonal. Upon further compression, the system exhibits a second first-order phase transition from a RHx state $\left(\phi=0.683, \Psi_{6}=\right.$ $\left.0.846, \Phi_{6}=0.185, X_{c}=-0.041\right)$ to an orientationally ordered chiral hexagonal (CHx) state $\left(\phi=0.710, \Psi_{6}=0.903, \Phi_{6}=0.733, X_{c}=\right.$ $0.843)$ at $p^{*} \sim 0.141$. This phase is shown in Figure 2 (a) and is characterised by large values of both order paramters $\Psi_{6}$ and $\Phi_{6}$, confirming the particles are aligned and arranged in an hexagonal lattice. Moreover, the large values of the chiral oder parameter $\left|X_{c}\right|$ reveal the platelets exhibit an offset angular displacement in a common direction. A close inspection of the $\mathrm{CHx}$ struc- 

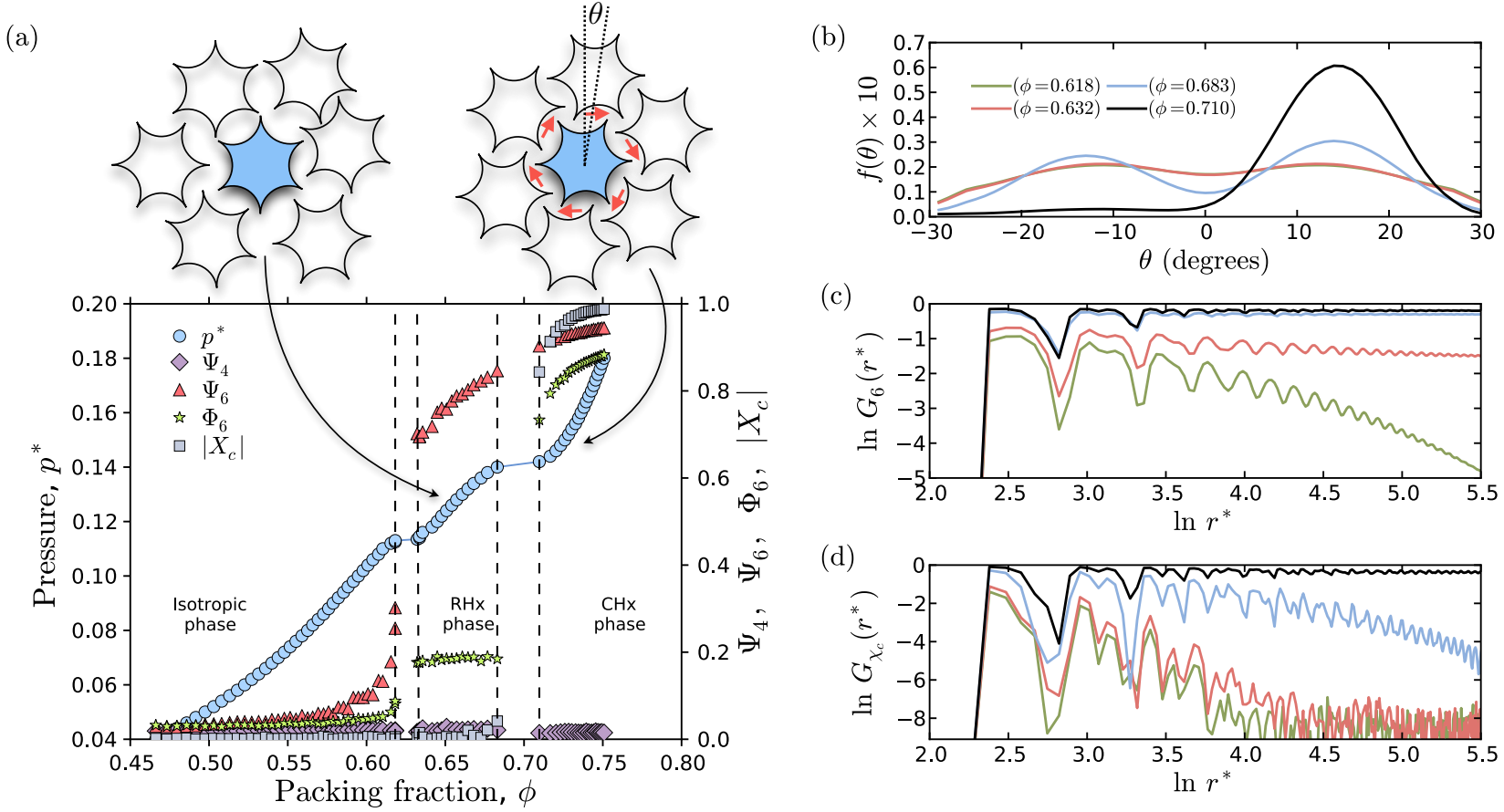

Fig. 1 (a) Pressure-packing fraction $\left(p^{*}-\phi\right)$ phase diagram for 1024 nonconvex hexagonal platelets with curvature $k=1 / 3$. The dependence of the global bond-order parameters $\Psi_{4}$ and $\Psi_{6}$, orientational order parameter $\Phi_{6}$, and chiral order parameter $X_{c}$ (in absolute numbers) on the packing fraction $\phi$ is also indicated. Statistical errors are smaller than the symbol sizes. Representative local configurations of the $\mathrm{RHx}$ and $\mathrm{CHx}$ phases are also shown on the top. (b) Probability distribution functions $f(\theta)$ of the angular displacement $\theta$ for nonconvex hexagonal platelets. The four curves correspond to the highest-density isotropic state $(\phi=0.618)$, (b) the lowest-density $\mathrm{RHx}$ state $(\phi=0.632)$, the highest-density $\mathrm{RHx}$ state $(\phi=0.683)$, and the lowest-density hexagonal chiral $(\mathrm{CHx})$ state $(\phi=0.710)$. In (c) and (d) the $G_{6}(r)$ and $G_{\chi_{c}}(r)$ correlations functions for the same states described in (b) using 9216 platelets are also shown.

ture shown in Figure 2(a) and at the top of Figure 1(a) reveals a clear offset clockwise angular displacement $\theta$ between two nearest edges of neighbouring particles to maximise their packing suggesting the system exhibits macrocopic CSB, i.e. this phase is a trichiral honeycomb structure ${ }^{34}$. The frequency distributions of angular displacements $f(\theta)$ are also used to analyse the CSB in hexagonal platelets. The results for $f(\theta)$ are shown in Figure 1(b) for four different states corresponding to the highestdensity isotropic state $(\phi=0.618)$, the lowest-density RHx state $(\phi=0.632)$, the highest-density RHx state $(\phi=0.683)$, and the lowest-density hexagonal chiral $(\mathrm{CHx})$ state $(\phi=0.710)$. The nearly flat distributions of $f(\theta)$ for the first two states reveal that the hexagonal platelets at these conditions are able to freely rotate exploring all different angular displacements. The highestdensity RHx state is still able to explore all values of $\theta$, however, $f(\theta)$ becomes clearly bimodal suggesting the particles forms clusters with local chirality with either clockwise or anticlockwise angular displacements, i.e. the system becomes racemic. For the lowest-density $\mathrm{CHx}$ state, the system becomes unimodal, with an intense peak centred at $\theta=15^{\circ}$, demonstrating that the chiral symmetry breaking is indeed macroscopic. This long-ranged macroscopic CSB is further confirmed by the behaviour of the correlation functions $G_{6}(r)$ and $G_{\chi_{c}}(r)$, defined as

$$
G_{6}(r)=\left\langle\bar{\psi}_{6}(0) \psi_{6}(r)\right\rangle,
$$

and

$$
G_{\chi_{c}}(r)=\left\langle\left|\chi_{c}(0) \chi_{c}(r)\right|\right\rangle,
$$

respectively, which are shown in Figures 1(c-d). It is evident from the behaviour of $G_{6}(r)$ that both RHx and CHx states exhibit long-range hexagonal order. However, only the $\mathrm{CHx}$ state exhibits long-range chiral order as revealed from the behaviour of $G_{\chi_{c}}(r)$, confirming the appearance of macroscopic CSB.

The phase behaviour of nonconvex polygons with 3,4 , and 5 -fold symmetries of curvature $k=1 / 3$ are also analysed to further investigate the role of symmetry on the formation of chiral structures. The results for the phase diagrams of these polygons are presented in Figures 3 (square platelets) and S1-S2 (pentagonal and triangular platelets) in the Supplementary Information $(\mathrm{SI})^{35}$. As one would expect, nonconvex squares, pentagons, and hexagons all form RHx phases at moderate packing fractions to maximise the orientational entropy. At higher densities, however, these particles self-assemble into distinct crystals that depend on their symmetry. The close-packed configurations of the different nonconvex polygons are shown in Figures 2(b-d). Nonconvex pentagons form a similar achiral ordered hexagonal phase as perfect pentagons do (see Figure 2(b)), which is characterised by alternating rows of oppositely aligned particles along one direction (striped hexagonal phase) ${ }^{32}$. Likewise, nonconvex triangles form an achiral striped triangular phase (see Figure 2(d)). Note that although perfect triangular platelets prefer to form structures with edge-to-edge alignment (triatic phase), nonconvex triangles 


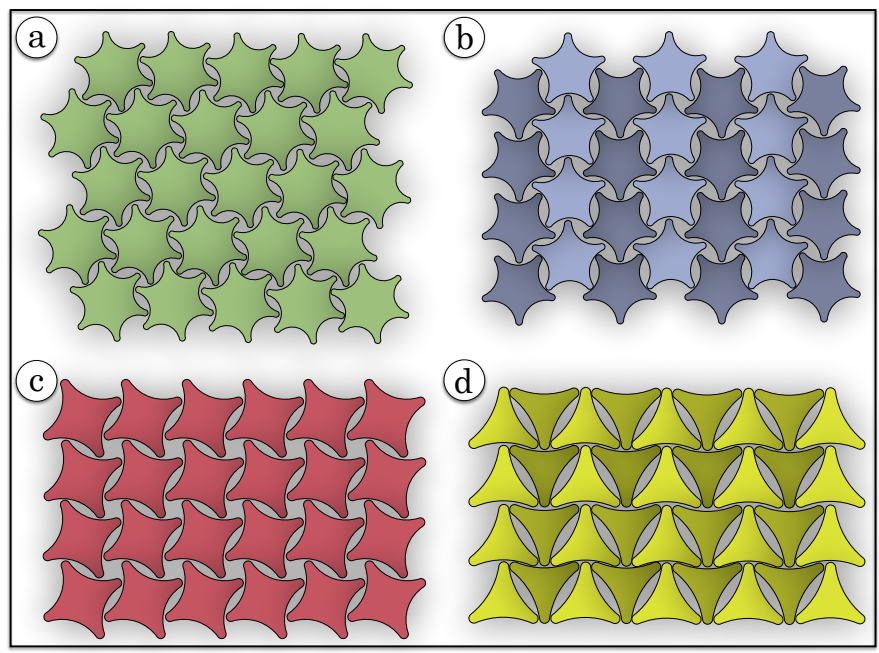

Fig. 2 Representative configurations of close-packed microstructures observed in monolayers of nonconvex platelets with curvature $k=1 / 3$ obtained from compression runs using NPT MD simulations. The structures agree with those obtained using the pressure-annealing method of Filion et al. ${ }^{27}$ The configurations correspond to (a) trichiral honeycomb $(\mathrm{CHx})$ structure formed by nonconvex hexagons, (b) striped hexagonal (vertex-to-edge) lattice formed by nonconvex pentagons, (c) tetrachiral (CSq) structure formed by non-convex squares, and (d) striped triangular lattice formed by nonconvex triangles. For (b) and (d), particles with opposite orientations are drawn in different shades.

pack more efficiently by arranging into alternating stripes with particles aligned vertex-to-edge ${ }^{36}$. In contrast, nonconvex square platelets exhibit a similar chiral behaviour as nonconvex hexagonal platelets and the results are presented in Figure 3. At sufficiently high densities, the system transforms from a RHx state $\left(\phi=0.642, \Psi_{4}=0.052, \Psi_{6}=0.664, \Phi_{4}=0.049, \chi_{c}=-0.004\right)$ to a tetrachiral (CSq) phase $\left(\phi=0.675, \Psi_{4}=0.917, \Psi_{6}=0.568, \Phi_{4}=\right.$ $\left.0.924, \chi_{c}=0.955\right)$ at $p^{*} \sim 0.465$. A representative configuration of this CSq structure is shown in Figure 2(c), where it is observed that particles exhibit an offset angular displacement in a common direction to maximise the interlocking of the curved edges thus producing a macroscopic CSB effect. Our results indicate that nonconvex polygons exhibit macroscopic CSB only when the number of nearest neighbours per particle in the condensed phases equals the symmetry of the particles promoting in this way a collective angular displacement in a common direction. In other words, only nonconvex hexagons and squares have suitable shapes to observe the amplification of the local chirality at high-densities to attain macroscopic CSB. It is important to stress that despite the propensity of nonconvex squares and hexagons to spontaneously induce macroscopic CSB, the system cannot control the handedness of the final crystal. Six repetitions of the compression runs for hexagonal platelets using different starting configurations renders equal amount of right and left handed $\mathrm{CHx}$ phases. This behaviour is a consequence of the lack of chirality in the shape of the individual particles.

The macroscopic CSB observed in nonconvex hexagons and squares, and the lack of in nonconvex pentagons and triangles, shares common features with the appearance of chiral structures observed during the buckling of $2 \mathrm{D}$ low-density cellular mate-

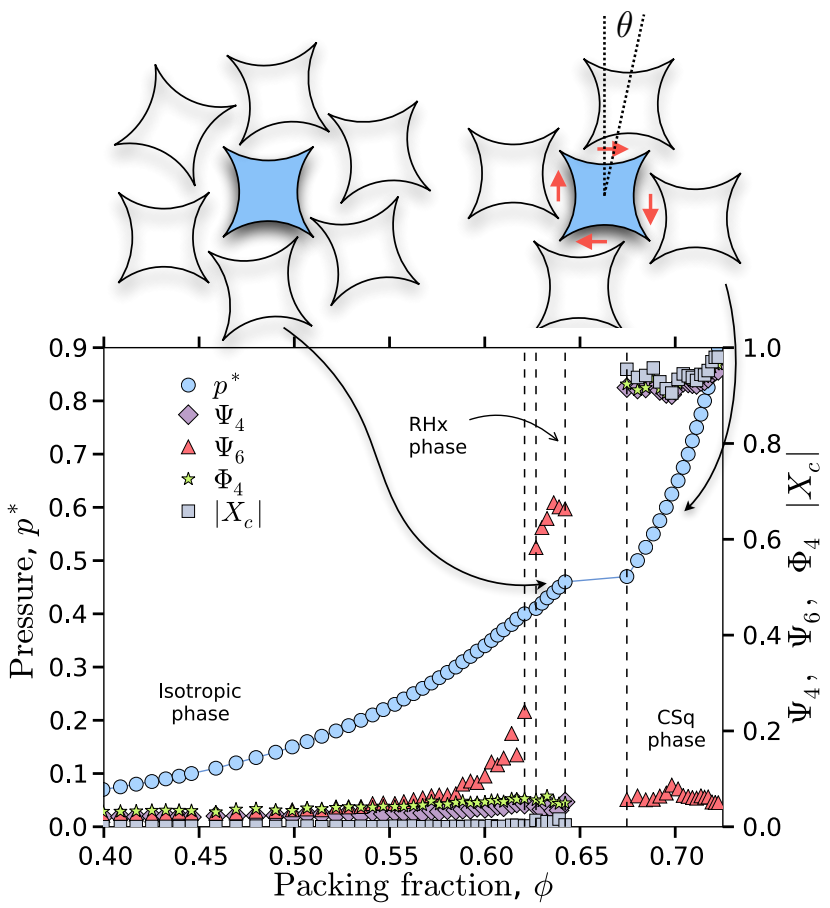

Fig. 3 (a) Pressure-packing fraction $\left(p^{*}-\phi\right)$ phase diagram for 1024 nonconvex square platelets with curvature $k=1 / 3$. The dependence of the global bond-order parameters $\Psi_{4}$ and $\Psi_{6}$, orientational order parameter $\Phi_{4}$, and chiral order parameter $X_{c}$ (in absolute numbers) on the packing fraction $\phi$ is also indicated. Statistical errors are smaller than the symbol sizes. Representative local configurations of the $\mathrm{RHx}$ and $\mathrm{CSq}$ phases are also shown on the top.

rials $^{34,37,38}$. Kang and co-workers ${ }^{37}$, for example, recently reported the appearance of chiral and achiral structures during the buckling of hexagonal and square honeycomb cellular materials. The swelling of the structural plates of the cellular materials promotes the formation of sinusoidal buckling instabilities (see Figure S4 in the $\mathrm{SI}^{35}$ ). In particular the authors observed that when the plates of the cellular materials exhibit half sinusoidal buckling patterns (either up $\frown$ or down $\smile$ ), the systems form achiral structures. However, buckling instabilities comprised of complete sinusoidal instabilities $(\sim$ for anticlockwise rotation or $\sim$ for clockwise rotation) induce all the walls of the cell to buckle with the same handness and macroscopic CSB is observed (see Figure $\mathrm{S} 4(\mathrm{~b}, \mathrm{~d})$ in the $\left.\mathrm{SI}^{35}\right)$. Although in our system there are no buckling instabilities as such, the edge-to-edge offset rotational shift between paired edges of neighbouring platelets induces a similar effect, i.e. the shift produces a complete sinusoidal-like effect ( $\propto$ for left-handed displacement or $\checkmark$ for right-handed displacement) as in the interlocking of mechanical gears. Therefore, only when all the edges of a polygon are able to interlock with the edges of neighbouring particles, the system can exhibit spontaneous macroscopic CSB. This is the case of nonconvex hexagonal and square platelets. A similar effect was observed in dodecagons. The close-packed structure of regular (convex) dodecagons is a hexagonal lattice in which 6 of the edges of the particles are in direct contact with the edges of the 6 nearest neighbours. In the case of nonconvex dodecagons, however, the same 6 curved 


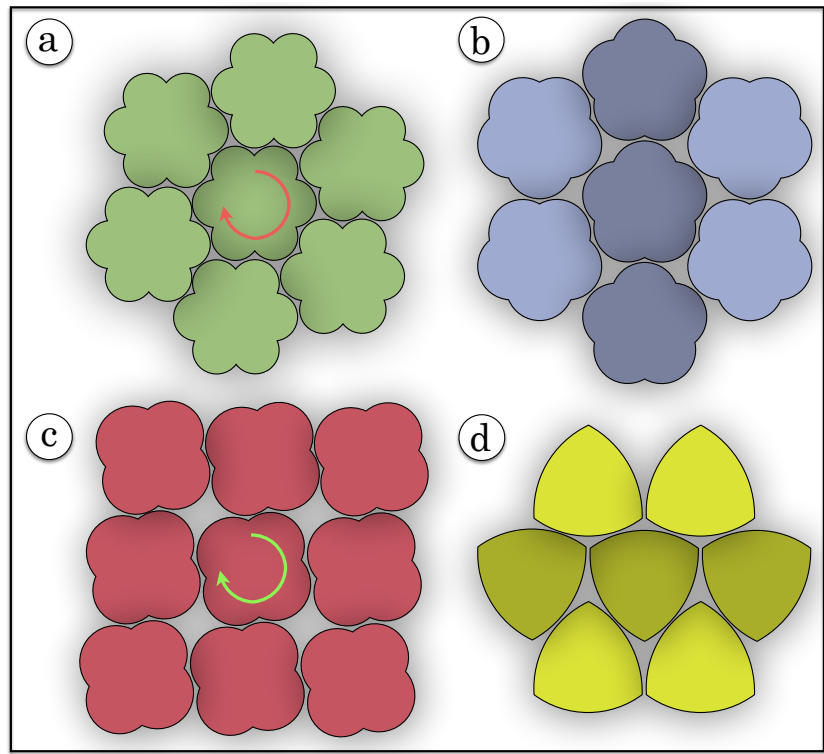

Fig. 4 Representative configurations of close-packed microstructures formed by nonconvex platelets with negative curvature $k$ (bumpy platelets). The configurations corresponds to crystals structures for (a) hexagons, (b) pentagons, (c) squares, and (d) triangles. Both hexagonal and square platelets exhibit the formation of chiral structures. Note that the parameter $k$ is different in each system and these arbitrary values were selected to show clearly the noconvex shape.

edges remained paired but now exhibiting an offset angular displacement in a common direction to maximise the packing of the system as shown in Figure S3(c) in the $\mathrm{SI}^{35}$. Based on this argument of edge's pairing, one would expect that both hexagonal and square platelets with negative curvature (negative values of $k$ leading to bumpy nonconvex hexagons and squares) should also exhibit macroscopic CSB since at high densities edges can be paired with either $\smile \frown$ or $\frown$ configurations as shown in Figure 4(a,c).

The same argument of pairing the edges in nonconvex polygons explains the lack of macroscopic CSB in nonconvex pentagons as it is impossible, by symmetry arguments, to pair all the edges of a pentagon with the edges of the nearest neighbours thus forbidding a uniform rotation in a single direction (see Figure $\mathrm{S} 5$ in the $\mathrm{SI}^{35}$ ). The same argument applies to polygons with symmetry higher than six and less than twelve. As mentioned before, nonconvex triangular platelets do not experience macroscopic CSB. Complete wavelength buckling instabilities, and even some particular cases of half wavelength instabilities ${ }^{38}$, in perfect triangular cellular materials can induce macroscopic CSB (see Figure S6(a)). However, nonconvex triangular platelets pack more efficiently in a a triangular lattice with vertex-to-edge (stripped) configuration that does not allow all the edges to be paired thus preventing macroscopic $\mathrm{CSB}^{36}$.

\section{Conclusions}

In conclusion, we have demonstrated that achiral nonconvex polygons with curved edges can form ordered crystalline structures exhibiting macroscopic chiral symmetry breaking driven by packing effects if the symmetry of the particles equals (or is mul- tiple of) the number of neighbours of the particles in the crystal. This is the situation of platelets with 4,6 , and 12 -fold symmetries. Chiral symmetry breaking is not observed in the case of triangles and pentagons as their symmetry precludes the formation chiral phases. Our results offer an straightforward strategy to form structured chiral surfaces by designing the shape of simple platelets that can be synthesised using existing experimental techniques such as soft lithography ${ }^{39,40}$. Since these nonconvex platelets cannot pack very efficiently due to the curved edges, these systems open the possibility of forming chiral crystal superlattices for applications in energy storage, electronics, and photonics ${ }^{41}$ when the polygons are mixed with spherical nanoparticles of suitable size that can fit in the interparticle voids. This hypothesis will be addressed in future work.

\section{Appendix. Particle model for athermal non- convex platelets}

The platelets are represented as a rigid planar body comprised of $N_{b}$ soft-repulsive spherical beads of diameter $\sigma$. Starting with a polygon with straight edges (see for example Figure 5(a) for the case of a triangle), the curvature of the platelet is controlled by shifting the beads of the edges of the original particle, originally separated by a distance $\sigma / 2$, towards the centre of the particle (keeping the beads forming the vertices fixed) according to the following quadratic function

$$
y(x ; n, k)=-\left[\frac{k}{3 \tan (\pi / n)} x^{2}+\frac{3(1-k)}{\tan (\pi / n)}\right],
$$

where $n$ is the symmetry of the platelets and $k$ is the degree of curvature. Note that the lengths in Equation 6 are given in units of $\sigma$. An example of a nonconvex platelet with 3-fold symmetry and curvature $k=1 / 3$ is shown in Figure 5(b) for illustration. Despite the platelets being modelled as rigid rings, the internal empty cavity of the particles is insignificant in two-dimensional systems. Therefore, the particles effectively represent nonconvex platelets of planar area $A_{p}$. This particle area $A_{p}$ is required to calculate the packing fraction of a system and is obtained according to the following expression:

$$
A_{p}=D_{n}(S, n)-n\left[E_{1}\left(N_{b, s}, n, k\right)+E_{2}(n)\right],
$$

where $D_{n}$ is the area of a straight polygon in which the platelet is inscribed, $N_{b, s}$ is the number of beads per side (fixed at $N_{b, s}=13$ for all cases presented in this study), and the regions $E_{1}$ and $E_{2}$ are defined in Figure 6. The area $E_{2}$ depends only on the number of sides of the platelet $n$ and is given by

$$
E_{2}(n)=\frac{1}{4} \tan ^{-1} \vartheta(n)-\frac{\pi}{4 n} .
$$

where $\vartheta(n)=(2 n)^{-1} \pi(n-2)$ is a half of the internal angle of the straight polygon shown in Figure 6. Finally, the expression for the area $E_{1}$ is obtained from integration of the quadratic function used to defined the curvature and is given by 

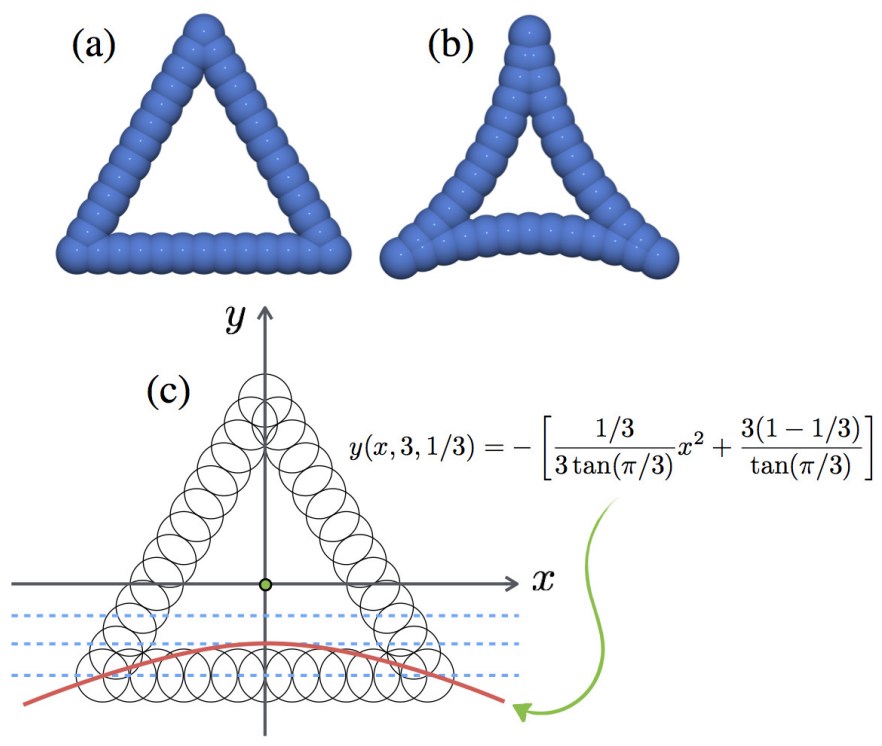

Fig. 5 Particle models of platelets using a collection of $N_{b}$ spherical beads interacting via the WCA potential (only the model for triangular platelets is shown). For all the platelets studied, 13 beads per side are used. Two different cases are shown: (a) Regular platelets with straight sides and (b) nonconvex platelets with curved edges. The curvature of platelets is controlled by shifting the positions of the beads towards the centre of the particle using a quadratic function shown in (c).

$$
\begin{aligned}
& E_{1}\left(N_{b, s}, n, k\right)=\frac{1}{8}\left(N_{b, s}-1\right)^{2} \tan \vartheta(n) \\
& -\left|\frac{1}{18}\left(N_{b, s}-1\right) \tan ^{-1}\left(\frac{\pi}{n}\right)\left[\frac{1}{16} k\left(N_{b, s}-1\right)^{2}+27(1-k)\right]\right|
\end{aligned}
$$

\section{Acknowledgements}

NP is very grateful to EPSRC and the University of Manchester for the Doctoral Training Award. We thank the Computational Shared Facility of the University of Manchester for computing time.

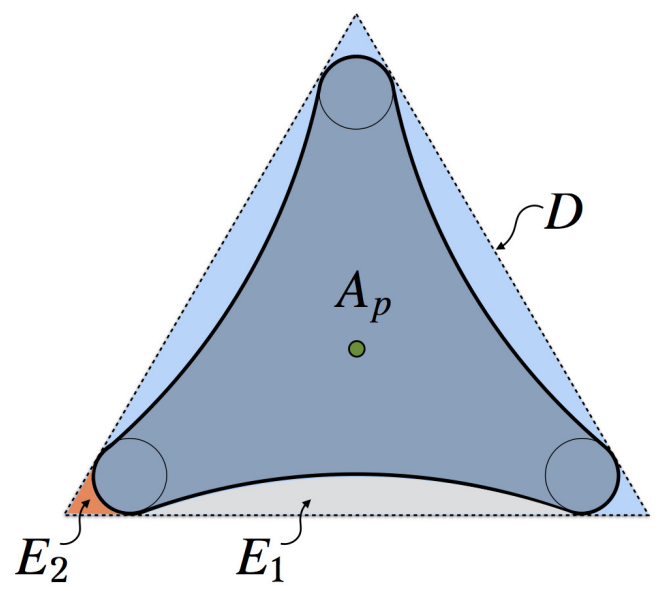

Fig. 6 Sketch of a triangular platelet with curvature $k$ showing the different regions used to calculate the area of the particle $A_{p}$.

\section{References}

1 L. Kelvin, J. Oxford Univ. Junior Scientific Club, 1894, 18, 3.

2 L. D. Barron, Nature, 2007, 446, 505-506.

3 M. Liu, L. Zhang and T. Wang, Chem. Rev., 2015, 115, 73047397.

4 M. M. Safont-Sempere, P. Osswald, M. Stolte, M. Grüne, M. Renz, M. Kaupp, K. Radacki, H. Braunschweig and F. Würthner, J. Amer. Chem. Soc., 2011, 133, 9580-9591.

5 M. Y. Mulla, E. Tuccori, M. Magliulo, G. Lattanzi, G. Palazzo, K. Persaud and L. Torsi, Nat. Commun., 2015, 6, 6010.

6 J. S. Seo, D. Whang, H. Lee, S. I. Jun, J. Oh, Y. J. Jeon and K. Kim, Nature, 2000, 404, 982-986.

7 R. E. Morris and X. Bu, Nat. Chem., 2010, 2, 353-361.

8 R. Zhu, X. N. Liu, G. K. Hu, C. T. Sun and G. L. Huang, Nat. Commun., 2014, 5, 5510.

9 W. Ma, H. Kuang, L. Wang, L. Xu, W.-S. Chang, H. Zhang, M. Sun, Y. Zhu, Y. Zhao, L. Liu, C. Xu, S. Link and N. A. Kotov, Sci. Rep., 2013, 3, 1934.

10 P. F. Damasceno, A. S. Karas, B. A. Schultz, M. Engel and S. C. Glotzer, Phys. Rev. Lett., 2015, 115, 158303.

11 S. Dussi and M. Dijkstra, Nat. Commun., 2016, 7, 11175.

12 K. Zhao, R. Bruinsma and T. G. Mason, Nat. Commun., 2012, 3, 801 .

13 S. P. Carmichael and M. S. Shell, J. Chem. Phys., 2013, 139, 164705.

14 K. Zhao and T. G. Mason, J. Phys. Condens. Matter, 2014, 26, 152101.

15 P.-Y. Wang and T. G. Mason, J. Amer. Chem. Soc., 2015, 137, 15308-15314.

16 D. R. Link, G. Natale, R. Shao, J. E. Maclennan, N. A. Clark, E. Körblova and D. M. Walba, Science, 1997, 278, 1924-1927.

17 C. Greco and A. Ferrarini, Phys. Rev. Lett., 2015, 115, 147801.

18 C. Avendaño and F. A. Escobedo, Curr. Opin. Colloid Interface Sci., 2017, 30, 62-69.

19 A. P. Gantapara, W. Qi and M. Dijkstra, Soft Matter, 2015, 11, 8684-8691.

20 M. Benedict and J. F. Maguire, Phys. Rev. B, 2004, 70, 174112.

21 J. D. Weeks, D. Chandler and H. C. Andersen, J. Chem. Phys., 1971, 54, 5237-5247.

22 C. Avendaño, G. Jackson, E. A. Müller and F. A. Escobedo, Proc. Natl. Acad. Sci. USA, 2016, 113, 9699-9703.

23 S. Plimpton, J. Comp. Phys., 1995, 117, 1-19.

24 W. G. Hoover, Phys. Rev. A, 1985, 31, 1695.

25 G. J. Martyna, M. L. Klein and M. Tuckerman, J. Chem. Phys., 1992, 97, 2635-2643.

26 G. J. Martyna, M. E. Tuckerman, D. J. Tobias and M. L. Klein, Mol. Phys., 1996, 87, 1117-1157.

27 L. Filion, M. Marechal, B. van Oorschot, D. Pelt, F. Smallenburg and M. Dijkstra, Phys. Rev. Lett., 2009, 103, 188302.

28 M. Parrinello and A. Rahman, J. Appl. Phys., 1981, 52, 71827190.

29 J. Gao and J. H. Weiner, J. Chem. Phys., 1989, 91, 3168.

30 D. R. Nelson and B. I. Halperin, Phys. Rev. B, 1979, 19, 2457- 
2484.

31 M. P. Allen and D. J. Tildesley, Computer Simulation of Liquids, Oxford University Press, Oxford, 1987.

32 T. Schilling, S. Pronk, B. Mulder and D. Frenkel, Phys. Rev. E, 2005, 71, 036138.

33 C. Avendaño and F. A. Escobedo, Soft Matter, 2012, 8, 46754681.

34 B. Haghpanah, J. Papadopoulos, D. Mousanezhad, H. NayebHashemi and A. Vaziri, Proc. R. Soc. A-Math. Phys. Eng. Sci., 2014, 470, 20130856.

35 See Supporting Information for more details.

36 S. Atkinson, Y. Jiao and S. Torquato, Phys. Rev. E, 2012, 86, 031302.

37 S. H. Kang, S. Shan, W. L. Noorduin, M. Khan, J. Aizenberg and K. Bertoldi, Adv. Mater., 2013, 25, 3380-3385.

38 S. H. Kang, S. Shan, A. Košmrlj, W. L. Noorduin, S. Shian, J. C. Weaver, D. R. Clarke and K. Bertoldi, Phys. Rev. Lett., 2014, 112, 098701.

39 C. J. Hernandez and T. G. Mason, J. Phys. Chem. C, 2007, 111, 4477-4480.

40 S.-M. Kang, C.-H. Choi, J. Kim, S.-J. Yeom, D. Lee, B. J. Park and C.-S. Lee, Soft Matter, 2016, 12, 5847-5853.

41 K. Hur, R. G. Hennig, F. A. Escobedo and U. Wiesner, Nano Lett., 2012, 12, 3218-3223. 


\title{
Supporting Information for: Macroscopic chiral symmetry breaking in monolayers of achiral nonconvex platelets
}

\author{
Nikoletta Pakalidou, ${ }^{1}$ David L. Cheung, ${ }^{2}$ Andrew J. Masters,${ }^{1}$ and Carlos Avendano ${ }^{1, *}$ \\ ${ }^{1}$ School Chemical Engineering and Analytical Science, \\ The University of Manchester, Sackville street, Manchester, M3 9PL, UK \\ ${ }^{2}$ School of Chemistry, National University of Ireland, Galway, University Road, Galway, Ireland
}

\section{S1. EQUATIONS OF STATE OF NONCONVEX POLYGONS}

In addition to the phase behaviour of nonconvex hexagonal and square platelets presented in the main text, we analyse the effect of the in-plane rotational symmetry of nonconvex polygons with three- and five-fold symmetries and curvature $k=1 / 3$. The phase diagrams for these systems are shown in Figures S1 and S2.

We discuss first the phase behaviour of nonconvex pentagons. The results, which are shown in Figure S1, are similar to the results previously reported for perfect pentagons with straight edges[1]. Nonconvex pentagons exhibit two phase transitions. The first transition is from an isotropic (Iso) state to a hexagonal rotator (RHx) phase in which the particles arrange in a hexagonal lattice with random orientations. The second transformation is from a RHx phase to a distorted hexagonal $(\mathrm{Hx})$ phase. In this $\mathrm{Hx}$ phase the five-fold orientational order parameter $\Phi_{5}$ is negligible for all packing fractions explored (results not shown), but $\Phi_{10}$ approaches to the unity which shows that the Hx phase is formed by alternating rows of oppositely pointing particles (see Figure 2(b) in the main text).

The results for nonconvex triangles are shown in Figure S2. Contrary to what it is observed in the simulations of perfect triangles studied by Gantapara and co-workers [2] where particles form a triatic phase, nonconvex triangular platelets with curvature $k=1 / 3$ exhibit a transition from a low-density isotropic phase to a striped (achiral) triangular (Tr) phase characterised by alternating rows of oppositely oriented particles (see Figure 2(d) in the main text).

Finally, based on the same symmetry arguments used for squares, pentagons, and hexagons, one would expect that non-convex platelets with 7-, 8-, 9-, 10-, and 11-fold symmetries cannot form chiral crystals as these structures form hexagonal crystals. This is indeed confirmed by analysing the closed-packed configurations of heptagons and octagons shown in Figure S3(a,b). The case of dodecagons, however, is different. Analysis of the closed-packed configurations of dodecagons with $k=1 / 3$ shown in Figure S3(c) reveal the formation of a chiral structure. In this case the symmetry of the platelets is a multiple of the number of nearest neighbours in the crystals thus half of the edges can be paired promoting a similar angular displacement as in the case of hexagons and squares.

\section{S2. GEOMETRICAL MECHANISM OF MACROSCOPIC CHIRAL SYMMETRY BREAKING.}

As shown in references [3-5], certain buckling instabilities in 2D low-density cellular materials can induce macroscopic CSB. These structures resemble the structures observed in nonconvex hexagons and squares. When these instabilities comprise only of half sinusoids, neither square nor hexagonal cellular materials exhibit macroscopic CSB (see Figures S4(a,c)). However, when the instabilities are comprised of complete sine waves, the system exhibits macroscopic CSB through the induction of a rotation of the walls of the cellular materials in the same direction (see $\mathrm{S} 4(\mathrm{~b}, \mathrm{~d}))$. In our systems of curved nonconvex platelets we do not have such buckling instabilities. However, the pairing of the curved edges of a given platelet with the edges of neighbouring particles induces a similar rotation mechanism as in cellular materials that leads to the appearance of macroscopic CSB. This induced rotation in nonconvex platelets is sketched in Figure S5.

In the case of triangular cellular materials, buckling instabilities can induce chiral rotation when the triangles are arranged in an edge-to-edge configuration as shown in Figure S6(a). However, the most efficient packing of nonconvex triangles is when they arrange in a vertex-to-edge configuration for which geometrical frustration prevents the rotation of the particles in the same direction as shown in Figure S6(b).

* carlos.avendano@manchester.ac.uk 


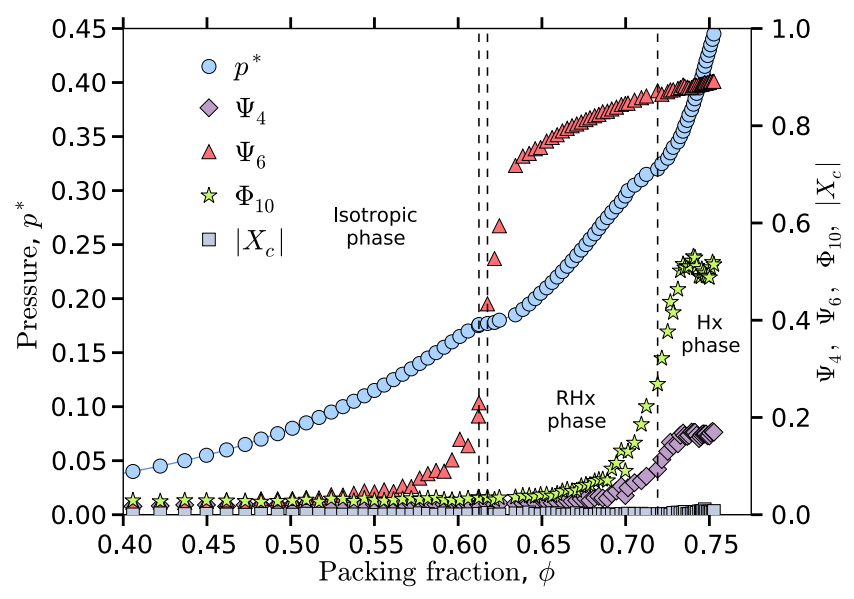

FIG. S1. Pressure-packing fraction $\left(p^{*}-\phi\right)$ phase diagram for nonconvex pentagon platelets with curvature $k=1 / 3$. The dependence of the global bond-order parameters $\Psi_{4}$ and $\Psi_{6}$, the orientational order parameter $\Phi_{10}$, and the chiral order parameter $X_{c}$ on the packing fraction $\phi$ is indicated.

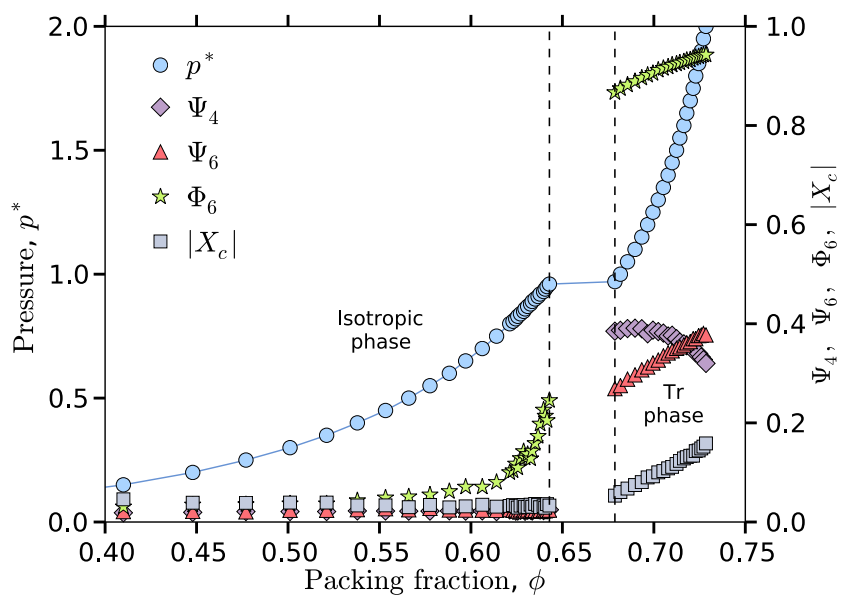

FIG. S2. Pressure-packing fraction $\left(p^{*}-\phi\right)$ phase diagram for nonconvex triangular platelets with curvature $k=1 / 3$. The dependence of the global bond-order parameters $\Psi_{4}$ and $\Psi_{6}$, the orientational order parameter $\Phi_{6}$, he chiral order parameter $X_{c}$ on the packing fraction $\phi$ is indicated. 


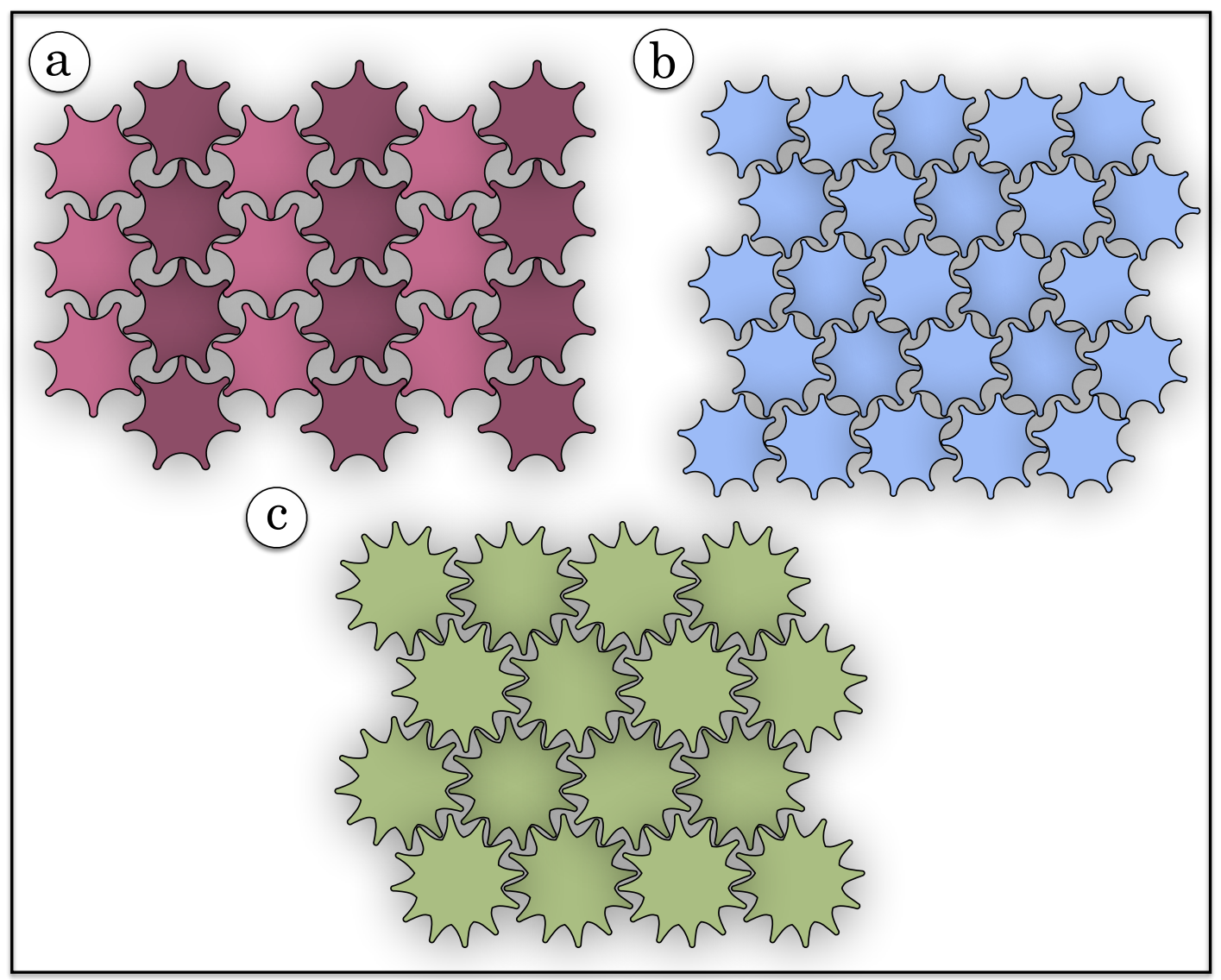

FIG. S3. Representative configurations of close-packed microstructures observed in monolayers formed by nonconvex platelets with curvature $k=-1 / 3$ obtained using the pressure-annealing method of Filion et al. [6] The configurations corresponds to crystals for (a) heptagons, (b) octagons, and (c) dodecagons. Only dodecagonal platelets exhibit the formation of a chiral structure.

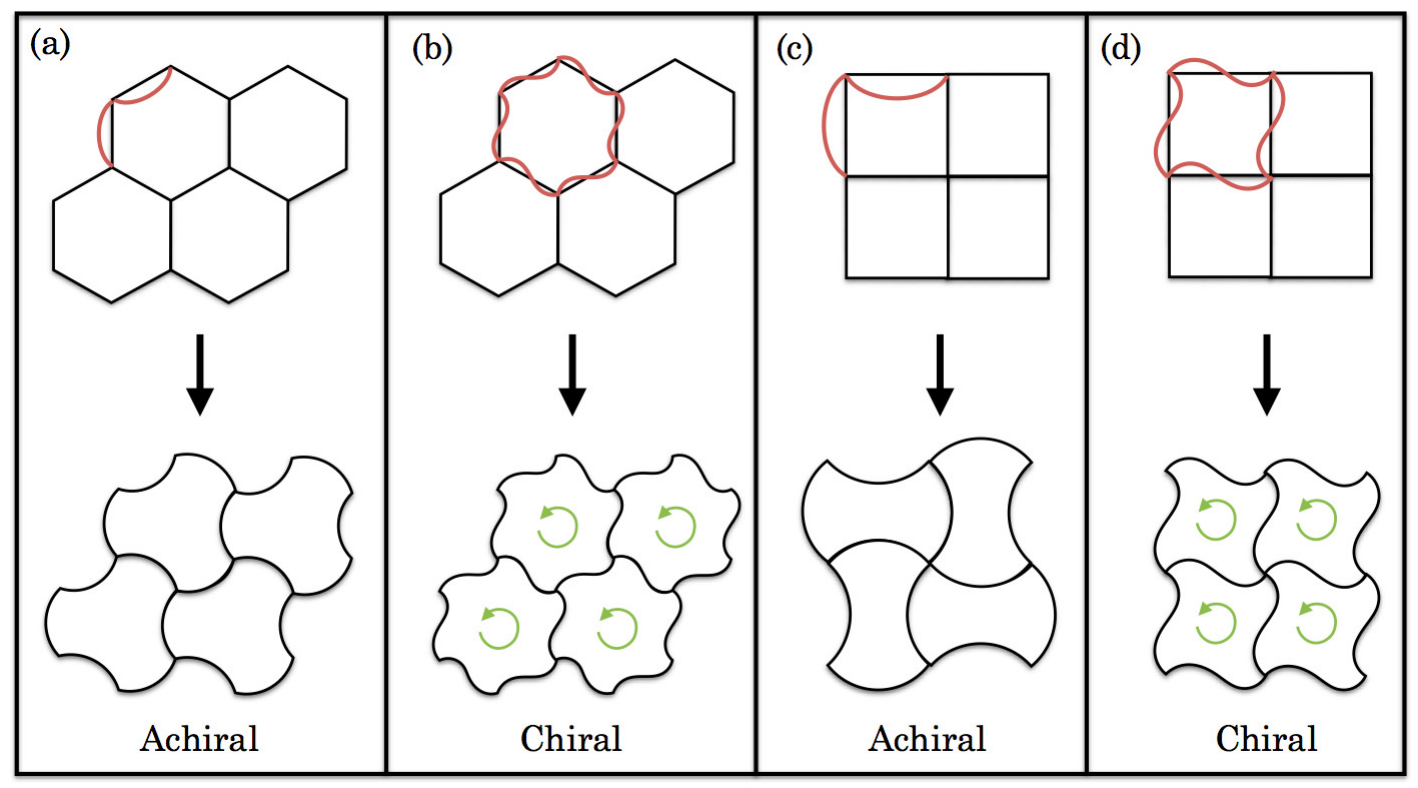

FIG. S4. Buckling instabilities in two-dimensional cellular materials with hexagonal (a,b) and square (c,d) symmetries. Figures (a) and (c) correspond to instabilities comprising half sinusoids showing the formation of achiral configurations. Figures (b) and (d) correspond to instabilities comprising a complete sinusoids that allows the structure to rotate in a common direction to form chiral configurations. 

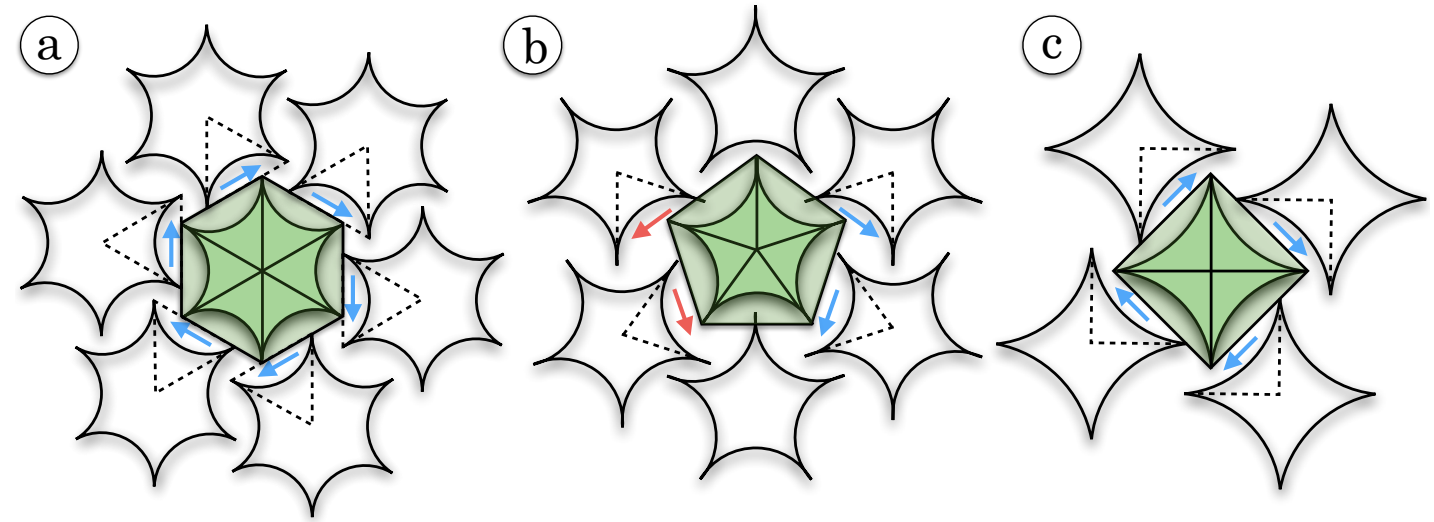

FIG. S5. Sketch of geometrical configurations in close-packed configuration of nonconvex polygonal platelets. The configurations correspond to (a) hexagons, (b) pentagons, and (c) square platelets. Only hexagonal and square platelets form configurations in which all the edges of a central particle can be paired with edges of neighbouring particles. This complete pairing of the edges allows a rotation of the system in a common direction allowing the formation of chiral phases. Conversely, pentagons exhibits geometrical frustration that forbids the rotation of the particles in a common direction.

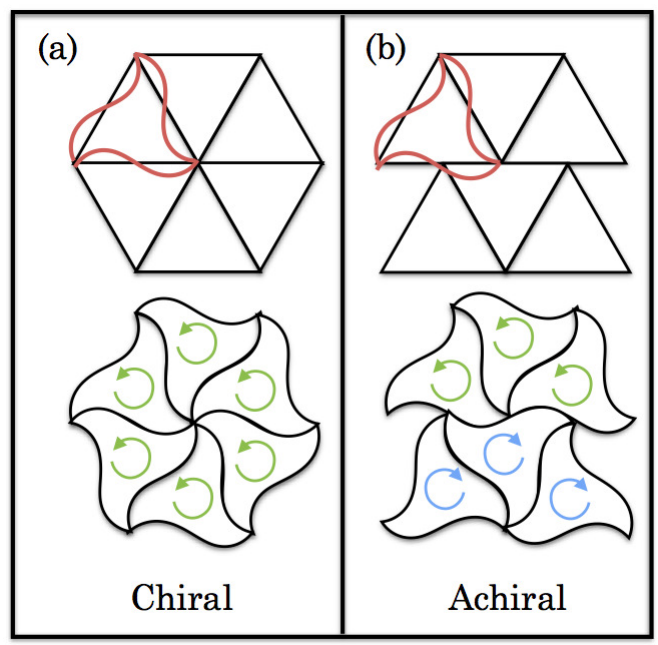

FIG. S6. Buckling instabilities in two-dimensional cellular materials with triangular geometry. Figure (a) corresponds to a edge-to-edge triangular structure, while Figure (b) corresponds to a vertex-to-edge triangular structure. Instabilities comprising of complete sine functions are shown for both systems, showing that only system (a) can form a chiral structure. The instabilities in structure (b) are geometrically frustrated. 
[1] T. Schilling, S. Pronk, B. Mulder, and D. Frenkel, Phys. Rev. E 71, 036138 (2005).

[2] A. P. Gantapara, W. Qi, and M. Dijkstra, Soft Matter 11, 8684 (2015).

[3] S. H. Kang, S. Shan, W. L. Noorduin, M. Khan, J. Aizenberg, and K. Bertoldi, Adv. Mater. 25, 3380 (2013).

[4] S. H. Kang, S. Shan, A. Košmrlj, W. L. Noorduin, S. Shian, J. C. Weaver, D. R. Clarke, and K. Bertoldi, Phys. Rev. Lett. 112, 098701 (2014).

[5] B. Haghpanah, J. Papadopoulos, D. Mousanezhad, H. Nayeb-Hashemi, and A. Vaziri, Proc. R. Soc. A-Math. Phys. Eng. Sci. 470, 20130856 (2014).

[6] L. Filion, M. Marechal, B. van Oorschot, D. Pelt, F. Smallenburg, and M. Dijkstra, Phys. Rev. Lett. 103,188302 (2009). 